\title{
NATURAL CONVECTION FLOW From A Porous Vertical Plate In Presence Of Heat Generation
}

\author{
Amena Ferdousi ${ }^{1}$ and M. A. Alim ${ }^{2}$ \\ ${ }^{1}$ Department of Electrical and Electronic Engineering \\ Eastern University, Dhaka, Bangladesh \\ ${ }^{2}$ Department of Mathematics \\ Bangladesh University of Engineering and Technology, Dhaka, Bangladesh \\ E-mail: amena@easternuni.edu.bd, maalim@math.buet.ac.bd.
}

\begin{abstract}
Natural convection flow from a porous vertical plate in presence of heat generation have been presented here. The governing boundary layer equations are first transformed into a non dimensional form and the resulting non linear system of partial differential equations are then solved numerically using finite difference method together with Keller-Box scheme. The numerical results of the surface shear stress in terms of skin friction coefficient and the rate of heat transfer in terms of local Nusselt number, velocity as well as temperature profiles are shown graphically and tabular form for a selection of parameters set of consisting of heat generation parameter $Q$, Prandtl number Pr.
\end{abstract}

Keywords: Porous plate, Heat generation, Natural convection.

\section{Introduction}

The study of heat generation or absorption in moving fluids is important in problems dealing with chemical reactions and those concerned with dissociating fluids. Possible heat generation effects may alter the temperature distribution; consequently the particle deposition rate in nuclear reactors, electronic chips and semiconductor wafers.

Free convection in presence of heat generation has been drawn forth not only for its fundamental aspects but also for its significance in the contexts of space technology and processes involving high temperature. In the presence of heat generation, natural convection boundary layer flow from a porous vertical plate of a steady two dimensional viscous incompressible fluid has been investigated. In this analysis consideration had been given to grey gases. Over the work it is assumed that the surface temperature of the porous vertical plate $T_{w}$, is constant, where $T_{w}>T_{\infty}$. Here $T_{\infty}$ is the ambient temperature of the fluid, $T$ is the temperature of the fluid in the boundary layer, $g$ is the acceleration due to gravity, the fluid is assumed to be a grey emitting and absorbing, but non scattering medium. In the present work variations in fluid properties are limited only to those density variations which affect the buoyancy terms.

Vajravelu and Hadjinicolaou [1] studied the heat transfer in a viscous fluid over a stretching sheet with viscous dissipation and internal heat generation. In their study, they considered that the volumetric rate of heat generation $q^{m}\left[\mathrm{~W} / \mathrm{m}^{3}\right]$ should be:

$q^{m}= \begin{cases}Q_{0}\left(T-T_{\infty}\right) & \text { for } T \geq T_{\infty} \\ 0 & \text { for } T<T_{\infty}\end{cases}$

where $Q_{0}$ is the heat generation constant. The above relation explained is valid as an approximation of the state of some exothermic process and having $T_{\infty}$ as the onset temperature. When the inlet temperature is not less than $T_{\infty}$ they used $Q_{0}\left(T-T_{\infty}\right)$. Merkin [2] studied free convection with blowing and suction. Lin and $\mathrm{Yu}$ [3] studied free convection on a horizontal plate with blowing and suction. Hossain et al [4] studied the effect of radiation on free convection flow with variable viscosity from a porous vertical plate. Hossain et al [5] studied flow of viscous incompressible fluid with temperature dependent viscosity and thermal conductivity past a permeable wedge with variable heat flux. Hossain and Takhar [6] studied radiation effect on mixed convection along a vertical plate with uniform surface temperature. Molla et al. [7] studied natural convection flow along a vertical wavy surface with uniform surface temperature in presence of heat 
generation/absorption. Akhter [8] studied the effect of radiations on free convection flow on sphere with isothermal surface and uniform heat flux. Ali [9] studied the effect of radiation on free convection flow on sphere with heat generation. Makinde and Moitsheki: [10] studied on non-perturbative techniques for thermal radiation effect on natural convection past a vertical plate embedded in a saturated porous medium. Makinde and Ogulu [11] studied the effect of thermal radiation on the heat and mass transfer flow of a variable viscosity fluid past a vertical porous plate permeated by a transverse magnetic field. Ogulu and Makinde [12] studied unsteady hydromagnetic free convection flow of a dissipative and radiating fluid past a vertical plate with constant heat flux. Hossain et al. [13] studied the effect of radiation on free convection flow from a porous vertical plate. They [13] analyzed a full numerical solution and found, an increase in Radiation parameter $R_{d}$ causes to thin the boundary layer and an increase in surface temperature parameter causes to thicken the boundary layer. The presence of suction ensures that its ultimate fate if vertically increased is a layer of constant thickness.

None of the aforementioned studies, considered the heat generation effects on laminar boundary layer flow of the fluids along porous plate without radiation heat loss.

The present study deals with natural convection flow from a porous vertical plate in presence of heat generation. The results will be obtained for different values of relevant physical parameters and will be shown in graphs as well as in tables.

The governing partial differential equations are reduced to locally non-similar partial differential forms by adopting some appropriate transformations. The transformed boundary layer equations are solved numerically using implicit finite difference scheme together with the Keller box technique [14]. Here, we have focused our attention on the evolution of the surface shear stress in terms of local skin friction and the rate of heat transfer in terms of local Nusselt number, velocity profiles as well as temperature profiles for selected values of parameters consisting of heat generation parameter $Q$, Prandtl number $P r$. In order to check the accuracy of our numerical results the present results are compared with [13].

\section{Formulation of the problem}

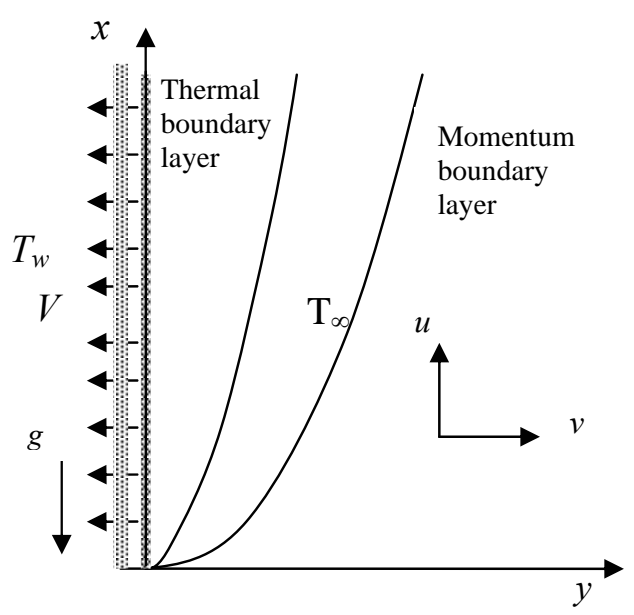

Fig. 1 The coordinate system and the physical model

We have investigated natural convection flow from a porous plate in presence of heat generation. The fluid is assumed to be a grey, emitting and absorbing. Over the work it is assumed that the surface temperature of the porous vertical plate, $T_{w}$, is constant, where $T_{w}>T$. The physical configuration considered is as shown in Fig. 1.

The conservation equations for the flow characterized with steady, laminar and two dimensional boundary layers; under the usual Boussinesq approximation, the continuity, momentum and energy equations can be written as:

$\frac{\partial u}{\partial x}+\frac{\partial v}{\partial y}=0$

$\rho\left(u \frac{\partial u}{\partial x}+v \frac{\partial u}{\partial y}\right)=\mu \frac{\partial^{2} u}{\partial x^{2}}+\rho g \beta\left(T-T_{\infty}\right)$

$\rho c_{p}\left(u \frac{\partial T}{\partial x}+v \frac{\partial T}{\partial y}\right)=k \frac{\partial^{2} T}{\partial y^{2}}+Q_{0}\left(T-T_{\infty}\right)$

With the boundary conditions

$x=0, y>0, u=0, T=T_{\infty}$.

$y=0, x>0, u=0, v=-V, T=T_{w}$

$y \rightarrow \infty, x>0, u=0, T=T_{\infty}$

where $\rho$ is the density, $k$ is the thermal conductivity, $\beta$ is the coefficient of thermal expansion, $v$ is the reference kinematic viscosity $v=\mu / \rho, \mu$ is the viscosity of the fluid, $C_{p}$ is the specific heat due to constant 
pressure. In order to reduce the complexity of the problem

Now introduce the following nondimensional variables:

$\eta=\frac{V y}{v \xi}, \xi=V\left\{\frac{4 x}{v^{2} g \beta \Delta T}\right\}^{\frac{1}{4}}$

$\psi=V^{-3} v^{2} g \beta \Delta T \xi^{3}\left\{f+\frac{\xi}{4}\right\}, \theta_{w}=\frac{T_{w}}{T_{\infty}}$,

Where, $\theta$ is the non-dimensional temperature function, $\theta_{\mathrm{w}}$ is the surface temperature parameter.

Substituting (5), (6) into Equations (1), (2) and (3) leads to the following nondimensional equations

$$
\begin{aligned}
& f^{\prime \prime \prime}+\theta-2 f^{\prime 2}+3 f f^{\prime \prime}+\xi f^{\prime \prime}=\xi\left(f^{\prime} \frac{\partial f^{\prime}}{\partial \xi} f^{\prime \prime} \frac{\partial f^{\prime}}{\partial \xi}\right) \\
& \frac{1}{p} \frac{\partial}{\partial \eta}\left[\frac{\partial \theta}{\partial \eta}\right]+Q \theta \xi+3 f \theta^{\prime}+\xi \theta^{\prime}=\xi\left(f^{\prime} \frac{\partial \theta}{\partial \xi} \frac{\partial}{\partial \xi} \theta^{\prime}\right)
\end{aligned}
$$

Where $\operatorname{Pr}=v C_{p} / k$ is the Prandtl number and $Q=v^{2} Q_{0} / V^{2} \rho C_{p}$ is the heat generation parameter. The boundary conditions (4) become

$$
\begin{aligned}
& f=0, f^{\prime}=0, \theta=1 \text { at } \eta=0 \\
& f^{\prime}=0, \theta=0 \text { as } \eta \rightarrow \infty
\end{aligned}
$$

The solution of equations (6), (8) enable us to calculate the nondimensional velocity components $\mathrm{u}, \overline{\mathrm{v}}$ from the following expressions

$$
\begin{aligned}
& \bar{u}=\frac{v^{2}}{V g \beta\left(T_{w}-T_{\infty}\right)} u=\xi^{2} f^{\prime}(\xi, \eta) \\
& \bar{v}=\frac{v}{V}=\xi^{-1}\left(3 f+\xi-\eta f^{\prime}+\xi \frac{\partial f}{\partial \xi}\right)
\end{aligned}
$$

In practical applications, the physical quantities of principle interest are the shearing stress $\tau_{\mathrm{w}}$ and the rate of heat transfer in terms of the skin-friction coefficients $C_{f x}$ and Nusselt number $N u_{x}$ respectively, which can be written as

$$
\begin{aligned}
& N u_{x}=\frac{v}{V \Delta T}\left(q_{c}\right)_{\eta=0}, C_{f}=\frac{V}{g \beta \Delta T}(\tau)_{\eta=0} \\
& \text { where } \tau_{w}=\mu\left(\frac{\partial u}{\partial y}\right)_{\eta=0} \text { and } q_{c}=-k\left(\frac{\partial T}{\partial y}\right)_{\eta=0}
\end{aligned}
$$

$q_{c}$ is the conduction heat flux.

Using the Equations (6) and the boundary condition (9) into (11) and (12), we get

$$
\begin{aligned}
& C_{f x}=\xi f^{\prime \prime}(x, 0) \\
& N u_{x}=-\xi^{-1} \theta^{\prime}(x, 0)
\end{aligned}
$$

The values of the velocity and temperature distribution are calculated respectively from the following relations:

$\bar{u}=\xi^{2} f^{\prime}(\xi, \eta), \quad \theta=\theta(x, y)$

\section{Method of Solution}

Solutions of the local non similar partial differential equation (7) to (8) subjected to the boundary condition (9) are obtained by using implicite finite difference method with Keller-Box Scheme, which has been described in details by Cebeci.[15]

The solution methodology of equations (7) and (8) with the boundary condition given in eqn. (9) for the entire $\xi$ values based on Keller - box scheme is proposed here. The scheme specifically incorporated a nodal distribution favoring the vicinity of the plate, enabling accuracy to be maintained in this region of steep gradient. In detail equations (7) and (8) are solved as a set of five simultaneous equations.

$f^{\prime \prime \prime}+3 f f^{\prime \prime}-2\left(f^{\prime}\right)^{2}+\theta-\xi f^{\prime \prime}$

$=\xi\left(f^{\prime} \frac{\partial f^{\prime}}{\partial \xi}-\frac{\partial f}{\partial \xi} f^{\prime \prime}\right)$

and

$\frac{1}{\operatorname{Pr}} \frac{\partial}{\partial \eta}\left[\frac{\partial \theta}{\partial \eta}\right]+Q \theta \xi^{2}+3 f \theta^{\prime}+\xi \theta^{\prime}$

$=\xi\left(f^{\prime} \frac{\partial \theta}{\partial \xi}-\frac{\partial f}{\partial \xi} \theta^{\prime}\right)$

To apply the aforementioned method, we first convert Equations (15)-(16) into the following system of first order equations with dependent variables $u(\xi, \eta), v(\xi, \eta)$, $p(\xi, \eta)$ and $g(\xi, \eta)$ as

$f^{\prime \prime}=u, u^{\prime}=v, \quad g=\theta$, and $\theta^{\prime}=p$

$v^{\prime}+p_{1} f v-p_{2} u^{2}+g-\xi v$

$=\xi\left(u \frac{\partial u}{\partial \xi}-\frac{\partial f}{\partial \xi} v\right)$

$\frac{1}{\operatorname{Pr}}\left[p^{\prime}\right]+p_{4} g \xi^{2}+\xi p+p_{1} f p$

$=\xi\left(u \frac{\partial g}{\partial \xi}-p \frac{\partial f}{\partial \xi}\right)$

where

$p_{1}=3, p_{2}=2, p_{4}=\mathrm{Q}$

The corresponding boundary conditions are $f(\xi, 0)=0, u(\xi, 0)=0$ and $g(\xi, 0)=0$

$u(\xi, \infty)=0, g(\xi, \infty)=0$ 
We now consider the net rectangle on the $(\xi, \eta)$ plane and denote the net point by

$$
\begin{aligned}
& \eta_{0}=0, \quad \eta_{j}=\eta_{j-1}+h_{j}, \quad j=1,2, \ldots J \\
& \xi^{0}=0, \quad \xi^{n}=\xi^{n-1}+k_{n}, \quad n=1,2, \ldots N
\end{aligned}
$$

We approximate the quantities $(f, u, v, p)$ at the points $\left(\xi^{n}, \eta_{j}\right)$ of the net by

$$
\left(f_{j}^{n}, u_{j}^{n}, v_{j}^{n}, p_{j}^{n}\right) \text { which we call net function. }
$$

$$
\begin{aligned}
& \eta_{j-1 / 2}=\frac{1}{2}\left(\eta_{j}-\eta_{j-1}\right) \\
& \xi^{n-1 / 2}=\frac{1}{2}\left(\xi^{n}+\xi^{n-1}\right) \\
& g_{j}^{n-1 / 2}=\frac{1}{2}\left(g_{j}{ }^{n}+g_{j}{ }^{n-1}\right) \\
& g_{j-1 / 2}^{n}=\frac{1}{2}\left(g_{j}{ }^{n}+g_{j-1}^{n}\right)
\end{aligned}
$$

Now we write the difference equations that are to approximate Equations (17) - (18) by considering one mesh rectangle for the mid point $\left(\xi^{n}, n_{j-1 / 2}\right)$ to obtain

$$
\begin{aligned}
& \frac{f_{j}^{n}-f_{j-1}^{n}}{h_{j}}=u_{j-1 / 2}^{n} \\
& \frac{u_{j}^{n}-u_{j-1}^{n}}{h_{j}}=v_{j-1 / 2}^{n} \\
& \frac{g_{j}^{n}-g_{j-1}^{n}}{h_{j}}=p_{j-1 / 2}^{n}
\end{aligned}
$$

Similarly Equations (18) - (19) are approximate by centering about the midpoint $\left(\xi^{n-1 / 2}, n_{j-1 / 2}\right)$. Centering the Equations (22) about the point $\left(\xi^{n-1 / 2}, n\right)$ without specifying $\eta$ to obtain the algebraic equations. The difference approximation to Equations (18)(19) become

$$
\begin{aligned}
& h_{j}^{-1}\left(v_{j}^{n}-v_{j-1}^{n}\right)+\left\{\left(p_{1}\right)_{j-1 / 2}^{n}+\alpha_{n}\right\}(f v)_{j-1 / 2}^{n} \\
& -\left\{\left(p_{2}\right)_{j-1 / 2}^{n}+\alpha_{n}\right\}\left(u^{2}\right)_{j-1 / 2}^{n}+g_{j-1 / 2}^{n} \\
& -(\xi v)_{j-1 / 2}^{n}+\alpha_{n}\left\{f_{j-1 / 2}^{n} v_{j-1 / 2}^{n-1}-v_{j-1 / 2}^{n} f_{j-1 / 2}^{n-1}\right\}=R_{j-1 / 2}^{n-1}
\end{aligned}
$$

where

$$
\begin{aligned}
L_{j-\frac{1}{2}}^{n-1} & =\left(p_{1}\right)_{j-\frac{1}{2}}^{n-1}(f v)_{j-\frac{1}{2}}^{n-1}-\left(p_{2}\right)_{j-\frac{1}{2}}^{n-1}\left(u^{2}\right)_{j-\frac{1}{2}}^{n-1}+g_{j-\frac{1}{2}}^{n-1} \\
& -(\xi p)_{j-\frac{1}{2}}^{n-1}+h_{j}^{-1}\left(v_{j}^{n-1}-v_{j-1}^{n-1}\right)
\end{aligned}
$$

And

$R_{j-\frac{1}{2}}^{n-1}=-L_{j-\frac{1}{2}}^{n-1}+\alpha_{n}\left\{-\left(u^{2}\right)_{j-\frac{1}{2}}^{n-1}+(f v)_{j-\frac{1}{2}}^{n-1}\right\}$

$\frac{1}{\operatorname{Pr}}\left[h_{j}^{-1}\left(p_{j}^{n}-p_{j-1}^{n}\right)+\xi_{j-1 / 2}^{n} p_{j-1 / 2}^{n}+\left(p_{4} \xi^{2}\right)_{j-1 / 2}^{n} g_{j-1 / 2}^{n}\right.$

$+\left(p_{1}\right)_{j-1 / 2}^{n}(f p)_{j-1 / 2}^{n}=-M_{j-1 / 2}^{n-1}+$

$\alpha_{n}\left[-(u g)_{j-1 / 2}^{n-1}+(f p)_{j-1 / 2}^{n-1}\right]+\alpha_{n}\left[(u g)_{j-1 / 2}^{n}\right.$

$\left.-(f p)_{j-1 / 2}^{n}-u_{j-1 / 2}^{n} g_{j-1 / 2}^{n-1}+u_{j-1 / 2}^{n-1} g_{j-1 / 2}^{n}\right\}$

$\left.+p_{j-1 / 2}^{n} f_{j-1 / 2}^{n-1}-p_{j-1 / 2}^{n-1} f_{j-1 / 2}^{n}\right]$

$\frac{1}{\operatorname{Pr}}\left[h_{j}^{-1}\left(p_{j}^{n}-p_{j-1}^{n}\right)+\xi_{j-1 / 2}^{n} p_{j-1 / 2}^{n}+\left(p_{4} \xi^{2}\right)_{j-1 / 2}^{n} g_{j-1 / 2}^{n}+\right.$

$\left\{\left(p_{1}\right)_{j-1 / 2}^{n}+\alpha_{n}\right\}(f p)_{j-1 / 2}^{n}-\alpha_{n}\left[\left\{(u g)_{j-1 / 2}^{n}\right.\right.$

$\left.-(u g)_{j-1 / 2}^{n-1}-u_{j-1 / 2}^{n} g_{j-1 / 2}^{n-1}+u_{j-1 / 2}^{n-1} g_{j-1 / 2}^{n}\right\}$

$\left.\left.+p_{j-1 / 2}^{n} f_{j-1 / 2}^{n-1}-p_{j-1 / 2}^{n-1} f_{j-1 / 2}^{n}\right\}\right]=T_{j-1 / 2}^{n-1}$

where

$M_{j-1 / 2}^{n-1}=\frac{1}{\operatorname{Pr}}\left[h_{j}^{-1}\left(p_{j}^{n-1}-p_{j-1}^{n-1}\right)-\left[\xi_{j-1 / 2}^{n-1} p_{j-1 / 2}^{n-1}\right.\right.$

$\left.+\left(p_{4} \xi_{2}\right)_{j-1 / 2}^{n-1} g_{j-1 / 2}^{n-1}+\left(p_{1}\right)_{j-1 / 2}^{n-1}(f p)_{j-1 / 2}^{n-1}\right]$

$T_{j-1 / 2}^{n-1}=-M_{j-1 / 2}^{n-1}+\alpha_{n}\left[(f p)_{j-1 / 2}^{n-1}-(u g)_{j-1 / 2}^{n-1}\right]$

The corresponding boundary conditions (21) become

$f_{0}^{n}=0, \quad u_{0}^{n}=0, \quad g_{0}^{n}=1$

$u_{J}^{n}=0, \quad g_{J}^{n}=0$

which just express the requirement for the boundary conditions to remain during the iteration process. Now we will convert the momentum and energy equations into system of linear Equations and together with the boundary conditions can be written in matrix or vector form, where the coefficient matrix has a block tri-diagonal structure. The whole procedure, namely reduction to first order followed by central difference approximations, Newton's quasi-linearization method and the block Thomas algorithm, is well known as the Keller- box method.

\section{Results and Discussions}

In this exertion natural convection flow on a porous vertical plate in presence of heat generation is investigated. Numerical values of local rate of heat transfer are calculated in terms of Nusselt number $N u_{x}$ for the surface of the porous vertical plate from lower 
stagnation point to upper stagnation point, for different values of the aforementioned parameters and these are shown in tabular form in Table: 1 and Table: 2 and graphically in Fiqure 2-5. The effect for different values of heat generation parameter $Q$ on local skin friction coefficient $C_{f x}$ and the local Nusselt number $N u_{x}$, as well as velocity and temperature profiles are displayed in Fig.2 and 5.These figures are to display how the profiles vary in $\xi$, the selected streetwise coordinate.

Figures 2(a)-2(b) display results for the velocity and temperature profiles, for different values of heat generation parameter $Q$ with Prandtl number $P r=1.0$ surface temperature parameter $\theta_{\mathrm{w}}=1.1$. It has been seen from Figures 2(a) and 2(b) that as the heat generation parameter $Q$ increases, the velocity and the temperature profiles increase. The changes of velocity profiles in the $\eta$ direction reveals the typical velocity profile for natural convection boundary layer flow, i.e., the velocity is zero at the boundary wall then the velocity increases to the peak value as $\eta$ increases and finally the velocity approaches to zero (the asymptotic value).

(a)
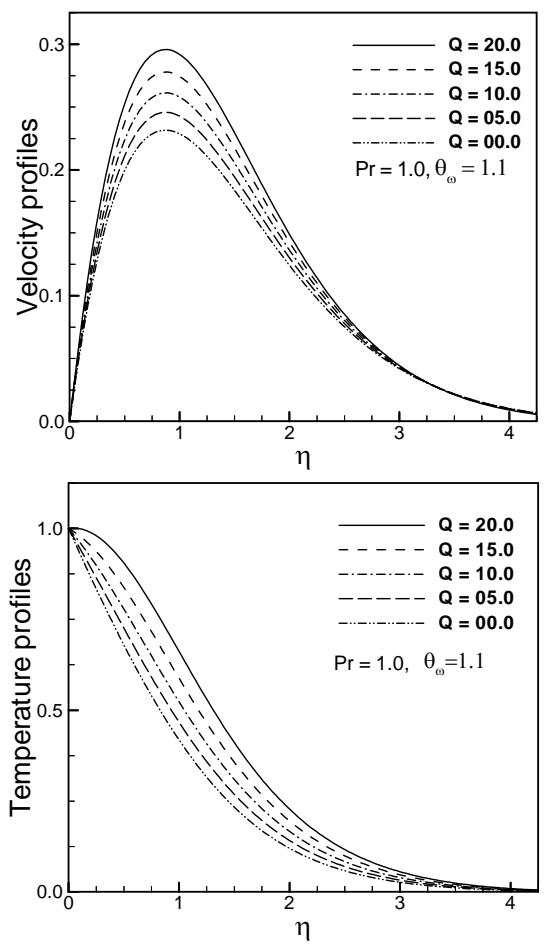

Fig. 2 (a) Velocity and (b) temperature profiles for different values of heat generation parameter $Q$ with others fixed parameters.
The maximum values of velocity are recorded to be 0.29587 at $\eta=0.88811$, 0.27770 at $\eta=0.83530,0.26129$ at $\eta=$ $0.88811,0.24586$ at $\eta=0.88811$ and 0.23163 at $\eta=0.88811$ for $Q=00.0,5.0,10.0,15.0$ and 20.0 respectively, the maximum values of velocity are recorded to be 0.29587 . Here, it is observed that at $\eta=0.88811$, the velocity increases by $27.73 \%$ as the heat generation parameter $Q$ changes from 20.0 to 0.0 . The changes of temperature profiles in the $\eta$ direction also shows the typical temperature profile for natural convection boundary layer flow that is the value of temperature profile is 1.0 (one) at the boundary wall then the temperature profile decreases gradually along $\eta$ direction for the values $\mathrm{Q}=0.0,5.0,10.0,15.0$. But for $\mathrm{Q}=$ 20.0 the temperature profile increases (at $\eta=$ 0.04001 temperature is 1.00106 for $\mathrm{Q}=20.0$ ) and again it decreases gradually along $\eta$ direction to the asymptotic value.

However, in Figures 3(a)-3(b), it is shown that when the Prandtl number $P r$ increases with $\theta_{\mathrm{w}}=1.1$ and $Q=1.0$, both the velocity and temperature profiles decrease.

(a)
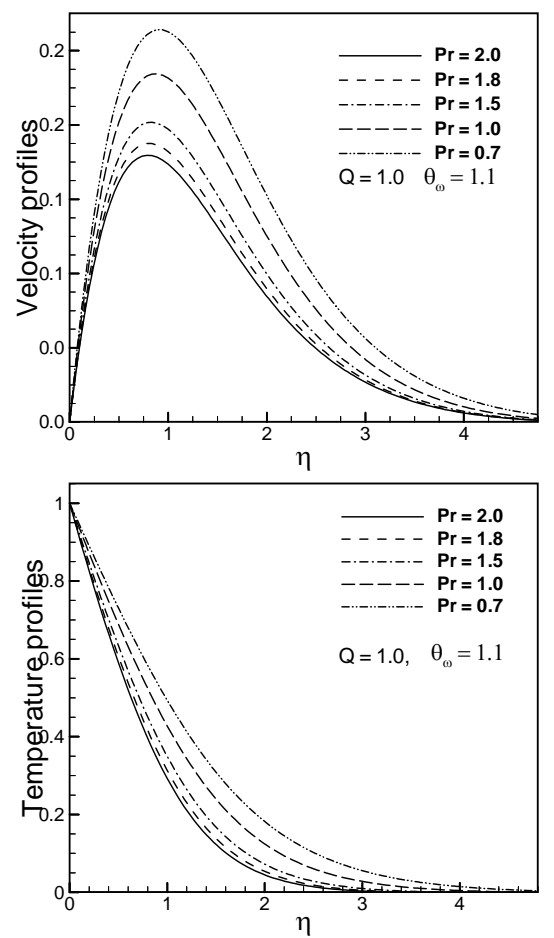

Fig. 3 (a) Velocity and (b) temperature profiles for different values of prandtl number $P r$ with others fixed parameters. 
Figures 4(a)-4(b) show that skin friction coefficient $C_{f x}$ increases and heat transfer coefficient $N u_{x}$ decrease respectively for increasing values of heat generation parameter $Q$. in case of Prandtl number $\operatorname{Pr}=$ 1.0 and surface temperature parameter $\theta_{\mathrm{w}}=$ 1.1. The values of skin friction coefficient $C_{f x}$ and Nusselt number $N u_{x}$ are recorded to be 0.16000, 0.26292, 0.14698, 0.14119, 0.13585 and $0.26292,1.10101,1.86750,2.57037$, 3.15576 for $Q=20.0,15.0,10.0,05.0$ and 00.0 respectively which occur at the same point $\xi=0.21$. Here, it is observed that at $\xi=$ 0.21 , the skin friction increases by $17.75 \%$ and Nusselt number $N u_{x}$ decreases by $91.66 \%$ as the heat generation parameter $Q$ changes from 00.0 to 20.0 .

The variation of the local skin friction coefficient $C_{f x}$ and local rate of heat transfer $N u_{x}$ for different values of Prandtl number $\mathrm{Pr}$ for $\theta_{\mathrm{w}}=1.1$ and $Q=1.0$ are shown in Figures 5(a)-5(b). We can observe from these figures that as the Prandtl number $P r$ increases, the skin friction coefficient decreases and rate of heat transfer increase.

(a)

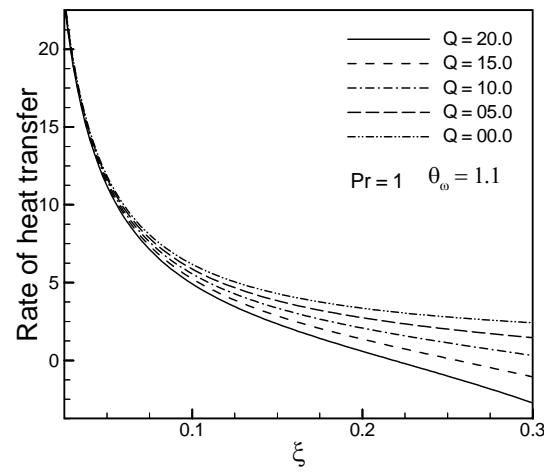

(b)

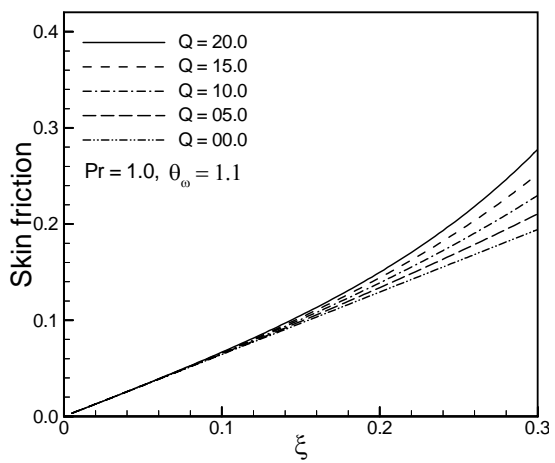

Fig. 4 (a) Skin friction and (b) rate of heat transfer for different values of heat generation parameter $\mathrm{Q}$ with others fixed parameters. (a)

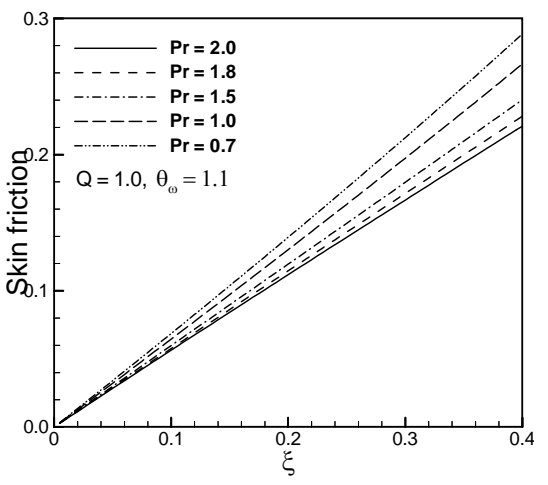

(b)

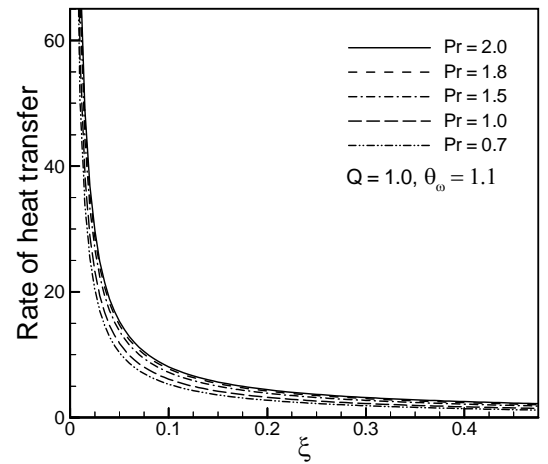

Fig. 5 (a) Skin friction and (b) rate of heat transfer for different values of Prandtl number parameter $P r$ with others fixed parameters.

Table 1 Skin friction coefficient and rate of heat transfer against $x$ for different values of heat generation parameter $Q$ with other controlling parameters $\operatorname{Pr}=1.0, R_{d}=0.1, \theta_{\mathrm{w}}=1.1$.

\begin{tabular}{|c|c|c|c|c|}
\hline \multirow{2}{*}{$\xi$} & \multicolumn{2}{|c|}{$Q=00.00$} & \multicolumn{2}{|c|}{$Q=05.00$} \\
\hline & $C_{f x}$ & $N u_{x}$ & $C_{f x}$ & $N u_{x}$ \\
\hline 0.01 & 0.00641 & 57.25668 & 0.00641 & 57.212612 \\
\hline 0.03 & 0.01926 & 19.42423 & 0.01928 & 19.32271 \\
\hline 0.05 & 0.03214 & 11.85422 & 0.03222 & 11.69491 \\
\hline 0.07 & 0.04505 & 8.61061 & 0.04524 & 8.39311 \\
\hline 0.09 & 0.05797 & 6.80953 & 0.05839 & 6.53330 \\
\hline 0.11 & 0.07092 & 5.66423 & 0.07167 & 5.32863 \\
\hline 0.13 & 0.08388 & 4.87206 & 0.08513 & 4.47636 \\
\hline 0.15 & 0.09686 & 4.29179 & 0.09878 & 3.83509 \\
\hline 0.17 & 0.10985 & 3.84865 & 0.11265 & 3.32983 \\
\hline 0.19 & 0.12284 & 3.49935 & 0.12678 & 2.91729 \\
\hline 0.21 & 0.13585 & 3.21706 & 0.14119 & 2.57037 \\
\hline \multirow{2}{*}{$\xi$} & \multicolumn{2}{|c|}{$Q=15.00$} & \multicolumn{2}{|c|}{$Q=20.00$} \\
\hline & $C_{f x}$ & $N u_{x}$ & $C_{f x}$ & $N u_{x}$ \\
\hline 0.01 & 0.00642 & 57.12444 & 0.00642 & 57.08034 \\
\hline 0.03 & 0.01931 & 19.11912 & 0.01933 & 19.01700 \\
\hline 0.05 & 0.03328 & 11.37400 & 0.03244 & 11.21239 \\
\hline 0.07 & 0.04564 & 7.95203 & 0.04585 & 7.72837 \\
\hline 0.09 & 0.05924 & 5.96815 & 0.05967 & 5.67901 \\
\hline 0.11 & 0.07323 & 4.63432 & 0.07404 & 4.27504 \\
\hline 0.13 & 0.08773 & 3.64650 & 0.08910 & 3.21108 \\
\hline 0.15 & 0.10285 & 2.86178 & 0.10501 & 2.34253 \\
\hline 0.17 & 0.11871 & 2.20351 & 0.12198 & 1.59093 \\
\hline 0.19 & 0.13545 & 1.62638 & 0.14022 & 0.90842 \\
\hline 0.21 & 0.15324 & 1.10101 & 0.16000 & 0.26292 \\
\hline
\end{tabular}

Numerical results of skin friction and rate of heat transfer are calculated from equation 
(13) for the surface of the porous plate from lower stagnation point to upper stagnation point at $\xi=0.01$ to $\xi=0.21$. Numerical values of $C_{f x}$ and $N u_{x}$ are depicted in Table 1. Here in this table the values of skin friction coefficient $C_{f x}$ and Nusselt number $N u_{x}$ are recorded to be $0.16000,0.26292,0.14698$, $0.14119,0.13585$ and $0.26292,1.10101$, $1.86750,2.57037,3.15576$ for $Q=20.0,15.0$, $10.0,05.0$ and 00.0 respectively which occur at the same point $\xi=0.21$. Here, it is observed that at $\xi=0.21$, the skin friction increases by $17.75 \%$ and Nusselt number $N u$ decreases by $91.66 \%$ as the heat generation parameter $Q$ changes from 20.0 to 00.0 .

\section{Comparison of the Results}

In order to verify the accuracy of the present work, the values of Nusselt number and skin friction for $Q=0, R_{d}=0.05 . P r=1.0$ and various surface temperature $\theta_{w}=1.1, \theta_{w}=2.5$ at different position of $\xi$ are compared with Hossain et al. [13] as presented in Table 2. The results are found to be in excellent agreement.

\begin{tabular}{|c|c|c|c|c|}
\hline \multicolumn{4}{|l}{ Table 2 Comparison of the present paper with } \\
Hossain et al. [13] \\
\hline \multirow{3}{*}{$\xi$} & \multicolumn{4}{|c|}{$\theta w=1.1$} \\
\cline { 2 - 5 } & \multicolumn{2}{|c|}{ Hossain } & \multicolumn{2}{c|}{ Hossain } \\
\cline { 2 - 5 } & $C_{f x}$ & $C_{f x}$ & $C_{f x}$ & $C_{f x}$ \\
\hline 0.1 & 0.0655 & 6.4627 & 0.06535 & 6.48306 \\
0.2 & 0.1316 & 3.4928 & 0.13138 & 3.50282 \\
0.4 & 0.2647 & 2.0229 & 0.26408 & 2.03018 \\
0.6 & 0.3963 & 1.5439 & 0.39519 & 1.55522 \\
0.8 & 0.5235 & 1.3247 & 0.52166 & 1.32959 \\
1.0 & 0.6429 & 1.1995 & 0.64024 & 1.20347 \\
1.5 & 0.8874 & 1.0574 & 0.88192 & 1.06109 \\
\hline \multirow{4}{*}{$\xi$} & \multicolumn{4}{|c|}{$\theta \mathrm{w}=2.5$} \\
\cline { 2 - 5 } & Hossain & \multicolumn{2}{c|}{ Hossain } \\
\cline { 2 - 5 } & Cfx & Cfx & Cfx & Cfx \\
\hline 0.1 & 0.0709 & 8.0844 & 0.07078 & 8.10360 \\
0.2 & 0.1433 & 4.2858 & 0.14313 & 4.29682 \\
0.4 & 0.2917 & 2.4003 & 0.29120 & 2.40669 \\
0.6 & 0.4423 & 1.7863 & 0.44145 & 1.78912 \\
0.8 & 0.5922 & 1.4860 & 0.59080 & 1.48991 \\
1.0 & 0.7379 & 1.1098 & 0.73590 & 1.31822 \\
1.5 & 1.0613 & 1.1098 & 1.05693 & 1.11262 \\
\hline
\end{tabular}

\section{Conclusion}

Natural convection flow on a porous vertical plate in presence of heat generation has been investigated for different values of relevant physical parameters including Prandtl number $P r$, and heat generation parameter $Q$.
- Significant effects of heat generation parameter $Q$ on velocity and temperature profiles as well as on skin friction and the rate of heat transfer have been found in this investigation but the effect of heat generation parameter $Q$ on rate of heat transfer is more significant. An increase in the values of heat generation parameter $Q$ leads to increase both the velocity and the temperature profiles, the local skin friction coefficient $C_{f x}$ increases at different position of $\eta$ and the local rate of heat transfer $N u_{x}$ decreases at different position of $\xi$ for $\xi$ $<0.1$ and decrease asymptotically when $\operatorname{Pr}=1.0$.

- $\quad$ The increase in Prandtl number Pr leads to decrease in all the velocity profile, the temperature profile, the local skin friction coefficient $C_{f x}$ but the local rate of heat transfer $N u_{x}$ increase.

\section{Nomenclatures}

$Q$ Heat generation parameter

$C_{f x}$ Local skin friction coefficient

$C_{p}$ Specific heat at constant pressure

$f$ Dimensionless stream function

$u$ Dimensionless velocity component along $x$-axis

$\mathrm{v}$ Dimensionless velocity component along $y$-axis

$g$ Acceleration due to gravity

$k$ Thermal conductivity

$N u_{x}$ Local Nusselt number

$T$ Temperature of the fluid in the boundary layer

$q_{w}$ Heat flux at the surface

$q_{c}$ Conduction heat flux.

$\mathrm{Pr}$ Prandtl number

$x \quad$ Axis in the direction along the surface

$y$ Axis in the direction normal to the surface

$T_{\infty}$ Temperature of the ambient fluid

$T_{w}$ Temperature at the surface

$V$ Wall suction velocity

\section{Greek symbols}

$\theta_{w} \quad$ Surface temperature parameter

$\beta$ Coefficient of thermal expansion

$\theta$ Dimensionless temperature function

$\xi$ Similarity variable

$\eta$ Similarity variable

$v$ Kinematic viscosity 
$\rho$ Density of the fluid

$\mu$ Viscosity of the fluid

$T$ Coefficient of skin friction

$\tau_{\mathrm{w}} \quad$ Shearing stress

$\psi$ Non-dimensional stream function

\section{Subscripts}

$w$ Wall conditions

$\infty$ Ambient temperature

\section{References}

[1] K. Vejravelu, A. Hadjinicolaou, "Heat transfer in a viscous fluid over a stretching sheet with viscous dissipation and internal heat generation". International Communications in Heat and Mass Transfer, Vol.20, 1993, pp 417-430.

[2] J. H. Merkin, "Free convection with blowing and suction”, International journal of heat and mass transfer, Vol.15, 1972, pp. 989-999.

[3] H. T. Lin, W. S. Yu, "Free convection on horizontal plate with blowing and suction", Transactions on ASME journal of Heat Transfer Vol. 110,1988, pp. 793-796.

[4] M. Anwar Hossain, Khalil Khanafer, Kambiz Khafai. "The effect of radiation on free convection flow with variable viscosity from a porous vertical plate", International Journal of Thermal science, Vol. 40, 1999, pp. 115-124.

[5] Md. Anwar Hossain, Md. Sazzad Munir, D. A. S. Rees, "Flow of viscous incompressible fluid with temperature dependent viscosity and thermal conductivity past a permeable wedge with variable heat flux", International Journal of Thermal science, Vol. 39, 2000, pp. 635-644.

[6] M. A. Hosain, H.S. Takhar, "Radiation effect on mixed convection along a vertical plate with uniform surface", Journal of Temperature, Heat and Mass Transfer, Vol. 31, 2001, pp.243-248.

[7] M. M. Molla, M. A. Hossain, L.S. Yao, "Natural convection flow along a vertical wavy surface with uniform surface temperature in presence of heat generation/absorption”, International
Journal of Thermal Science, Vol. 43, 2004, pp.157-163.

[8] Tahmina Akhter, "Effect of Radiation on Natural Convection Flow on a Sphere with Isothermal surface and uniform Heat Flux", M.Phil Thesis, Department of Mathematics, Bangladesh University of Engineering and Technology (BUET), Dhaka, Bangladesh, 2007.

[9] Md. Miraj Ali, "Numerical Study of Radiation on Natural Convection Flow on a Sphere with Heat Generation”, M.Phil Thesis, Department of Mathematics, Bangladesh University of Engineering and Technology (BUET), Dhaka, Bangladesh, 2007.

[10] O. D. Makinde, R. J. Moitsheki, "On nonperturbative techniques for thermal radiation effect on natural convection past a vertical plate embedded in a saturated porous medium", Mathematical Problems in Engineering, Vol. 2008, 689074 (11pp), 2008.

[11] O. D. Makinde, A. Ogulu, "The effect of thermal radiation on the heat and mass transfer flow of a variable viscosity fluid past a vertical porous plate permeated by a transverse magnetic field", Chemical Engineering Communications, Vol. 195, No. 12, 2008, pp. 1575 -1584.

[12] A. Ogulu, O. D. Makinde, "Unsteady hydromagnetic free convection flow of a dissipative and radiating fluid past a vertical plate with constant heat flux", Chemical Engineering Communications Vol. 196, No. 4, 2009, pp. 454-462.

[13] M. A. Hossain, M. A. Alim, D. A. S. Rees, "The effect of radiation on free convection flow from a porous vertical plate", International Journal of Heat and Mass Transfer, Vol. 42, 1999, pp. 81-91.

[14] H.B. Keller, "Numerical methods in boundary layer theory", Annual Review of Fluid Mechanics, Vol. 10, 1978, pp. 417-433.

[15] T. Cebeci, P. Bradshaw, "Physical and Computational Aspects of Convective Heat Transfer”, Springer, New York, 1984. 